\title{
Dynamic Cell Association for Downlink Sum Rate Maximization in Multi-Cell Heterogeneous Networks
}

\author{
Steven Corroy*, Laetitia Falconetti** and Rudolf Mathar* \\ ${ }^{*}$ Institute for Theoretical Information Technology, RWTH Aachen University, Aachen, Germany \\ **Ericsson Research, Aachen, Germany \\ \{corroy, mathar\}@ti.rwth-aachen.de, laetitia.falconetti@ericsson.com
}

\begin{abstract}
In this work, we consider a heterogeneous network consisting in several macro nodes and pico nodes. Our goal is to associate users, belonging to this network, to one of the nodes, while maximizing the sum rate of all users. We also want to analyze the load balancing achieved by this association. Therefore, we develop a new theoretical framework to study cell association for the downlink of multi-cell networks and derive an upper bound on the achievable sum rate. We propose a dynamic cell association heuristic, which achieves performance close to optimal. Finally, we verify our results through numerical evaluations and implement the proposed heuristic in an LTE simulator to demonstrate its viability.
\end{abstract}

\section{INTRODUCTION}

Heterogeneous Network (HetNet) is seen as a key component of cellular networks to meet the increasing demand for mobile broadband traffic. The considered HetNet consists of nodes operating on the same frequency band and using different Transmit (Tx) powers. The macro nodes ensure the basic coverage while the pico nodes provide higher capacity in areas with high user equipment (UE) density. The considered pico nodes use the same frequency band as the macro nodes, but have a much smaller transmit (Tx) power.

A fundamental problem in a cellular network is to associate UEs with a serving node. In current systems as in 3GPP Long Term Evolution (LTE), this problem is solved using a simple algorithm. A UE is associated with the node from which it receives the highest power. We call this algorithm the best SNR heuristic. It has the advantage to be robust against interference. However, since the macro nodes transmit with a higher power than the pico nodes, only a small fraction of UEs is associated to pico nodes [1]. This results in a strong load imbalance between macro and pico nodes, limiting the user throughput.

To cope with this drawback, a new method has been proposed within 3GPP [1], [2]. It consists in artificially increasing the power received by UEs from pico nodes by adding a fixed bias to it. We call this algorithm the fixed range heuristic. This algorithm enables more UEs to connect to pico nodes, yielding a better balanced load among the macro and pico nodes, and a larger average rate. However, due to the bias, certain UEs are connected to the pico node while they receive a stronger signal from the macro node and the improved load balancing may not always compensate the degradation in Signal to Interference and Noise Ratio (SINR) that these UEs encounter.

This work was partly supported by the UMIC research cluster of the RWTH Aachen University.
Our goal is 1) to determine a dynamic scheme for cell association that maximizes the sum rate of all UEs and 2) to understand the trade-off between the load balancing, in terms of user share between the nodes, and the interference level.

There has been some work in the field of cell association for load balancing in 3G networks. In [3], a centralized algorithm is proposed for a scenario in which the base stations (BSs) serve a single UE at each time slot. In [4] and [5], the optimal cell association for a proportional fair scheduler is derived by solving a sequence of Boolean linear programs.

In contrast to the present work, [3]-[5] do not consider a HetNet scenario, where there is a large power imbalance between nodes and a specific structure of the interference. Further we analyze the trade-off between the received interference and the load balancing instead of optimizing directly the load balancing. Finally, the dynamic cell association that we propose has a complexity linear in the number of UEs.

The present work contains numerous contributions. We propose a new theoretical framework enabling to analyze dynamic cell association in a multi-cell HetNet and derive an upper bound on the achievable downlink sum rate using convex optimization. We propose a heuristic with a low complexity coming close to the upper bound. We validate our theoretical results for a simple setup through numerical evaluations and implement our heuristic in an LTE simulator to analyze its behavior in a more realistic system.

Section II and III describe our optimization problem. Section IV derives an upper bound on the solution of this problem and a heuristic approaching this bound. Section V and VI present numerical results and Section VII concludes this work.

\section{DYNAMIC CELL ASSOCIATION WITHIN A TIGHTLY COORDINATED CELL CLUSTER}

In the considered HetNet, the macro nodes provide the basic coverage, whereas pico nodes are placed in the coverage area of each macro node to enhance capacity at these locations. The dynamic cell association is carried out within a cluster of coordinated nodes $\mathcal{S}$. The cluster $\mathcal{S}$ contains $m$ macro cell geographical areas $\mathcal{A}_{j}$. Each $\mathcal{A}_{j}$ includes one macro node and one or more pico nodes. The dynamic cell association relies on the best SNR heuristic to determine the group of active users $\mathcal{U}_{j}$ that falls under the responsibility of the nodes from any $\mathcal{A}_{j}$. The dynamic cell association is then applied to know which node from $\mathcal{A}_{j}$ will serve which users from $\mathcal{U}_{j}$. Fig. 1 illustrates this scenario. 


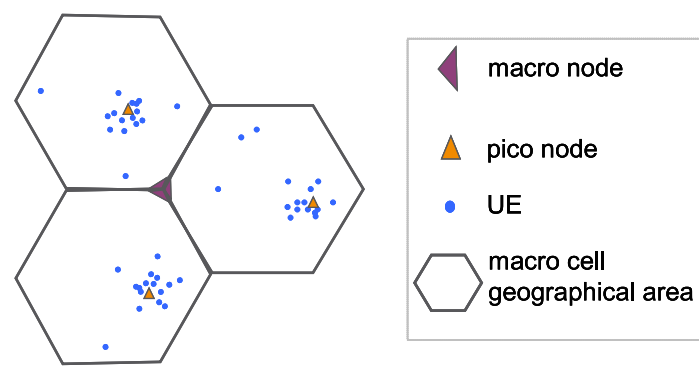

Fig. 1. Scenario with tightly coordinated macro and pico nodes.

The cell association and the resource scheduling are performed on two different frequency basis. The OFDM resources must be allocated for each time transmission interval (TTI). The cell association however only depends on long-term signal measurements and the number of active UEs in the system. A new association only takes place when UEs terminate or start a transmission or when the long-term measurements have significantly changed.

To upper bound the achievable sum rate of the network, it will be advantageous to decouple the association problem and the scheduling problem. In the present work we assume that a node shares its bandwidth equally among all its associated UEs. It corresponds to a Round Robin scheduler. This approach extends easily to another scheduler, e.g., proportional fairness, by simply scaling the UE rates with a weight proportional to their past rate.

An important requirement for dynamic cell association is that nodes are tightly coordinated to exchange efficiently information and react quickly when the network status has changed. It may also be preferable that the different nodes appear to the user as the same cell, as described in [6]. This makes the handover invisible to UEs, enables to shorten the handover delay and avoid the usual handover signaling overhead. In that case the cell association is actually a point selection since, for the UEs, there is only one cell. We assume in this work that all nodes are tightly coordinated.

\section{SYSTEM MODEL AND OPTIMIZATION PROBLEM}

We consider a cooperation cluster $\mathcal{S}$ with $m$ macro cell geographical areas $\mathcal{A}_{j}$ containing each one macro node and one pico node. The macro node of $\mathcal{A}_{j}$ has a transmit power $\sigma_{M_{j}}$ while the pico node of $\mathcal{A}_{j}$ uses a lower transmit power $\sigma_{P_{j}} \leq \sigma_{M_{j}}, j=1, \ldots, m$. All nodes operate with a bandwidth $B$ at the same frequency band. We assume that $\mathcal{U}_{j}$ contains $n$ UEs for which a cell association should be defined (there are $m n$ UEs in $\mathcal{S}$ ). A UE in $\mathcal{U}_{j}$ must be associated to either the macro node or the pico node of $\mathcal{A}_{j}$.

Consider $\mathbf{x}_{M_{j}}, \mathbf{x}_{P_{j}} \in\{0,1\}^{n}$, representing the association of the UEs in the macro cell area $\mathcal{A}_{j}$, defined as follows,

- $x_{M_{j}, k}=1$ and $x_{P_{j}, k}=0$, if UE $k \in \mathcal{U}_{j}$ is associated with the macro node.

- $x_{M_{j}, k}=0$ and $x_{P_{j}, k}=1$, if UE $k \in \mathcal{U}_{j}$ is associated with the pico node.

Since each UE of $\mathcal{U}_{j}$ is associated with a single node, $\mathbf{x}_{M_{j}}+$ $\mathbf{x}_{P_{j}}=\mathbf{1}$, where $\mathbf{1}$ is a vector of size $n$ containing only '1's.
The goal of the present work is to maximize the function $f\left(\mathbf{x}_{1}, \ldots, \mathbf{x}_{j}, \ldots, \mathbf{x}_{m}\right)$, where $\mathbf{x}_{j} \triangleq\left[\mathbf{x}_{M_{j}}^{\mathrm{T}} \mathbf{x}_{P_{j}}^{\mathrm{T}}\right]^{\mathrm{T}}$ and $f$ represents the sum rate of all $m n$ users. To get an estimate of the achievable user rate with a certain cell association, the equation $\mathrm{C}(\gamma)=\log _{2}(1+\gamma)$ is used. $\gamma$ denotes the user SINR calculated based on the average channel gain, since the cell association mechanism should be valid over a long term.

We denote $g_{M_{i}, j k}$ and $g_{P_{i}, j k}$ as the average channel gains between a UE $k$ in $\mathcal{U}_{j}$ and the macro and pico node $i$ respectively. Correspondingly, the average received power from the macro and pico node $i$ at UE $k$ in $\mathcal{U}_{j}$ is given by $\hat{\sigma}_{M_{i}, j k}=\sigma_{M_{i}} g_{M_{i}, j k}$ and $\hat{\sigma}_{P_{i}, j k}=\sigma_{P_{i}} g_{M_{i}, j k}$ respectively. Further we define $\sigma_{n}$ as the noise and interference power at the receiver measured over $B$. The interference component of $\sigma_{n}$ is an estimate of the interference power received from all nodes outside the coordination cluster $\mathcal{S}$.

We assume that a node always allocates all available frequency resources to its associated UEs at a given time. In other words, as soon as there is one active UE associated with a node, this node creates interference over the whole bandwidth.

We now define two vectors $\mathbf{y}_{M}, \mathbf{y}_{P} \in\{0,1\}^{m}$ defined as follows, $y_{M, j}=1$ if there is at least one UE associated to the macro node $j$ and $y_{M, j}=0$ otherwise. Similarly $y_{P, j}=1$ if the pico node $j$ is the serving node of at least one UE and $y_{P, j}=0$ otherwise. Note that $\mathbf{y}_{M}$ and $\mathbf{y}_{P}$ are completely defined by the vectors $\mathbf{x}_{M_{j}}$ and $\mathbf{x}_{P_{j}}$. The introduction of $\mathbf{y}_{M}$ and $\mathbf{y}_{P}$ is the key enabling us later to break up a hard problem into smaller ones for which we can find an upper bound.

The achievable rate $r_{M_{j}, k}$ achieved by a user $k \in \mathcal{U}_{j}$ in the downlink, if it is the only UE associated with the macro node $j$, is given by

$r_{M_{j}, k}=B \cdot \mathbf{C}\left(\hat{\sigma}_{M_{j}, j k} /\left(\sum_{i=1, i \neq j}^{m} y_{M, i} \hat{\sigma}_{M_{i}, j k}+\sum_{i=1}^{m} y_{P, i} \hat{\sigma}_{P_{i}, j k}+\sigma_{n}\right)\right)$.

Similarly, if UE $k$ is the only UE associated with the pico node $j$, its downlink achievable rate $r_{P_{j}, k}$ is given by

$r_{P_{j}, k}=B \cdot \mathbf{C}\left(\hat{\sigma}_{P_{j}, j k} /\left(\sum_{i=1}^{m} y_{M, i} \hat{\sigma}_{M_{i}, j k}+\sum_{i=1, i \neq j}^{m} y_{P, i} \hat{\sigma}_{P_{i}, j k}+\sigma_{n}\right)\right)$.

Finally we define $\mathbf{r}_{M_{j}} \triangleq\left[\begin{array}{ll}r_{M_{j}, 1}, \ldots, r_{M_{j}, m} & \mathbf{0}^{\mathrm{T}}\end{array}\right]^{\mathrm{T}}$, $\mathbf{r}_{P_{j}} \triangleq\left[\begin{array}{ll}\mathbf{0}^{\mathrm{T}} & r_{P_{j}, 1}, \ldots, r_{P_{j}, m}\end{array}\right]^{\mathrm{T}}, \mathbf{1}_{1} \triangleq\left[\begin{array}{ll}\mathbf{1}^{\mathrm{T}} & \mathbf{0}^{\mathrm{T}}\end{array}\right]^{\mathrm{T}}$ and $\mathbf{1}_{2} \triangleq$ $\left[\begin{array}{ll}\mathbf{0}^{\mathrm{T}} & \mathbf{1}^{\mathrm{T}}\end{array}\right]^{\mathrm{T}}$, where $\mathbf{0}$ is a vector of size $n$ containing only ' 0 's.

We now formulate the problem of finding the cell association, which maximizes the sum rate of all UEs as follows.

$$
\begin{array}{cl}
\underset{\mathbf{x}_{1}, \ldots, \mathbf{x}_{m}}{\operatorname{maximize}} & \sum_{j=1}^{m} \frac{\mathbf{r}_{M_{j}}^{\mathrm{T}} \mathbf{x}_{j}}{\mathbf{1}_{1}^{\mathrm{T}} \mathbf{x}_{j}}+\frac{\mathbf{r}_{P_{j}}^{\mathrm{T}} \mathbf{x}_{j}}{\mathbf{1}_{2}^{\mathrm{T}} \mathbf{x}_{j}} \\
\text { subject to } & {[\mathbf{I} \mathbf{I}] \mathbf{x}_{j}=\mathbf{1}} \\
& x_{j k} \in\{0,1\}, k=1, \ldots, 2 n,
\end{array}
$$

where $\mathbf{I}$ is the identity matrix of size $n$.

This is important to model the fact that the achievable rate for a UE decreases when more UEs are associated with the same node. A discussed in Section II, to model a Round Robin 
scheduler, we assume that all nodes distribute an equal share of the time-frequency resources among the served users. This is reflected in problem (3), by the division by $\mathbf{1}_{1}^{\mathrm{T}} \mathbf{x}_{j}$ and $\mathbf{1}_{2}^{\mathrm{T}} \mathbf{x}_{j}$.

Note that in this problem, both $\mathbf{r}_{M_{j}}$ and $\mathbf{r}_{P_{j}}$ depend on $\mathbf{y}_{M}$ and $\mathbf{y}_{P}$, which themselves depend on $\mathbf{x}_{j}$ in a non-linear and non-convex manner. At first glance this problem might look very hard to approach.

\section{UPPER BOUND FOR SUM RATE MAXIMIZATION}

In this section an upper bound for the optimal value of Problem (3) is derived. Furthermore, a simple heuristic producing a solution close to the optimum is described.

\section{A. Problem decomposition}

The main idea to find an upper bound on the solution of Problem (3) is to break up this complicated problem in a sequence of smaller ones. The key step is to go over all possible combinations of $\mathbf{y}_{M}$ and $\mathbf{y}_{P}$. In our problem, for each macro cell area $\mathcal{A}_{j}$, only the following cases may occur.

1) $y_{M, j}=1$ and $y_{P, j}=0$, i.e., all users in $\mathcal{U}_{j}$ are associated with the macro node of $\mathcal{A}_{j}$. In this case the user association is directly given, namely $\mathbf{x}_{j}=\left[\begin{array}{ll}\mathbf{1}^{\mathrm{T}} & \mathbf{0}^{\mathrm{T}}\end{array}\right]^{\mathrm{T}}$. The interference created towards a user $k$ from a different macro cell area $i \neq j$, is given by $\hat{\sigma}_{M_{j}, i k}$.

2) $y_{M, j}=0$ and $y_{P, j}=1$, i.e., all users in $\mathcal{U}_{j}$ are associated with the pico node of $\mathcal{A}_{j}$. The user association is $\mathbf{x}_{j}=\left[\begin{array}{ll}\mathbf{0}^{\mathrm{T}} & \mathbf{1}^{\mathrm{T}}\end{array}\right]^{\mathrm{T}}$. The interference created towards a user $k$ from a different macro cell area $i \neq j$ is given by $\hat{\sigma}_{P_{j}, i k}$.

3) $y_{M, j}=1$ and $y_{P, j}=1$, i.e., the users in $\mathcal{U}_{j}$ are distributed between the macro and the pico nodes of $\mathcal{A}_{j}$. In that case we need to find the optimal association for users in $\mathcal{U}_{j}$. The interference created towards a user $k$ from a different macro cell area $i \neq j$ is given by $\hat{\sigma}_{M_{j}, i k}+\hat{\sigma}_{P_{j}, i k}$.

The first important point to understand is that if $\mathbf{y}_{M}$ and $\mathbf{y}_{P}$ are fixed and known, then each node knows the interference coming from the other nodes of the other macro cell areas. Consequently, if we know which macro and pico nodes are active, each term of the sum in Problem (3) is independent of the others (since now all $\mathbf{r}_{M_{j}}$ and $\mathbf{r}_{P_{j}}$ are constant). Therefore we can maximize each term of the sum, $\frac{\mathbf{r}_{M_{j}}^{\mathrm{T}} \mathbf{x}_{j}}{\mathbf{1}_{1}^{\mathrm{T}} \mathbf{x}_{j}}+\frac{\mathbf{r}_{P_{j}}^{\mathrm{T}} \mathbf{x}_{j}}{\mathbf{1}_{2}^{\mathrm{T}} \mathbf{x}_{j}}$, independently of each other, for all possible combinations of $y_{M, j}$ and $y_{P, j}$. Since the association is known for the cases when either $y_{M, j}=0$ or $y_{P, j}=0$, the corresponding sum rate within $\mathcal{A}_{j}$ can be calculated directly. If $y_{M, j}=1$ and $y_{P, j}=1$, we need to find an upper bound on the optimal value of Problem (3), for a fixed value of $\mathbf{y}_{M}$ and $\mathbf{y}_{P}$.

\section{B. Complexity of the bounding algorithm}

The second important point is the evaluation of the complexity of this method. For each macro cell area $\mathcal{A}_{j}$, we have three cases to evaluate, which makes a total of $3^{m}$ combinations. Of course this approach is not feasible for a large coordination set $\mathcal{S}$ with many macro cell areas. But for the most realistic cases with smaller coordination set (say with $m \leq 10$ ) we transform the problem of finding the best association among $2^{m n}$ possibilities (for $m=3$ and $n=10$, it means $2^{30}$ possibilities) into $3^{m}$ small problems (for $m=3$ and $n=10$, 27 problems).

\section{Problem relaxation}

It remains to find the best association for case 3 , i.e., when $y_{M, j}=1$ and $y_{P, j}=1$. For a given macro cell area $\mathcal{A}_{j}$ and given a specific combination of $\mathbf{y}_{M}$ and $\mathbf{y}_{P}$, we need to solve the following problem

$$
\begin{array}{cl}
\underset{\mathbf{x}_{j}}{\operatorname{maximize}} & \frac{\mathbf{r}_{M_{j}}^{\mathrm{T}} \mathbf{x}_{j}}{\mathbf{1}_{1}^{\mathrm{T}} \mathbf{x}_{j}}+\frac{\mathbf{r}_{P_{j}}^{\mathrm{T}} \mathbf{x}_{j}}{\mathbf{1}_{2}^{\mathrm{T}} \mathbf{x}_{j}} \\
\text { subject to } & {[\mathbf{I} \mathbf{I}] \mathbf{x}_{j}=\mathbf{1}, \mathbf{1}_{1}^{\mathrm{T}} \mathbf{x}_{j} \geq 1, \mathbf{1}_{2}^{\mathrm{T}} \mathbf{x}_{j} \geq 1} \\
& x_{j k} \in\{0,1\}, k=1, \ldots, 2 n,
\end{array}
$$

where the second and third constraints enforce the fact that at least one UE is associated to the macro and pico node. We can find an upper bound on the solution of Problem (4) by proceeding to the following steps.

- We make the variable change $\mathbf{X} \triangleq \mathbf{x}_{j} \mathbf{x}_{j}^{\mathrm{T}}$, which, among others, transforms the constraint $x_{j k} \in\{0,1\}$ into $X_{j k} \in\{0,1\}$.

- Since the constraint $\mathbf{X}=\mathbf{x}_{j} \mathbf{x}_{j}^{\mathrm{T}}$ is equivalent to the two constraints $\mathbf{X} \succcurlyeq \mathbf{0}$ and $\operatorname{rank}(\mathbf{X})=1$, we exchange these constraints.

- We relax the nonconvex constraints by changing $X_{j k} \in\{0,1\}$ to $0 \leq X_{j k} \leq 1$ and dropping the rank constraint $\operatorname{rank}(\mathbf{X})=1$.

- We explicitly rewrite constraints on the structure of the problem which disappeared by dropping the rank constraint. Namely we know that $\sum_{j=1}^{2 n} \sum_{k=1}^{2 n} X_{j k}=n^{2}$ since $\mathbf{x}_{j}$ had exactly $n$ entries equal to 1 and the other equal to 0 . Furthermore we know that we have at least $n-1$ entries equal to 1 in the upper right corner of $\mathbf{X}$ (equal to $\mathbf{x}_{M_{j}} \mathbf{x}_{P_{j}}^{\mathrm{T}}$ ) since at least one UE is associated to the macro and pico node.

These steps enable us to find an upper bound on the solution of Problem (4) by solving the following problem

$$
\begin{array}{cl}
\underset{\mathbf{X}}{\operatorname{maximize}} & \frac{\mathbf{r}_{M_{j}}^{\mathrm{T}} \mathbf{X} \mathbf{1}_{2}+\mathbf{r}_{P_{j}}^{\mathrm{T}} \mathbf{X} \mathbf{1}_{1}}{\mathbf{1}_{1}^{\mathrm{T}} \mathbf{X} \mathbf{1}_{2}} \\
\text { subject to } & {[\mathbf{I} \mathbf{I}] \operatorname{diag}(\mathbf{X})=\mathbf{1}} \\
& \mathbf{1}_{1}^{\mathrm{T}} \operatorname{diag}(\mathbf{X}) \geq 1, \mathbf{1}_{2}^{\mathrm{T}} \operatorname{diag}(\mathbf{X}) \geq 1 \\
& 0 \leq X_{j k} \leq 1 j, k=1, \ldots, 2 n, \mathbf{X} \succcurlyeq \mathbf{0} \\
& \sum_{j=1}^{2 n} \sum_{k=1}^{2 n} X_{j k}=n^{2}, \sum_{j=1}^{n} \sum_{k=n+1}^{2 n} X_{j k} \geq n-1 .
\end{array}
$$

Problem (5) is quasiconcave [7] since all its constraints are convex and its objective function is quasiconcave. To see this, observe that all superlevel sets of the objective function,

$$
\mathcal{S}_{t}=\left\{\mathbf{X} \mid \mathbf{1}_{1}^{\mathrm{T}} \mathbf{X} \mathbf{1}_{2}>0, \mathbf{r}_{M_{j}}^{\mathrm{T}} \mathbf{X} \mathbf{1}_{2}+\mathbf{r}_{P_{j}}^{\mathrm{T}} \mathbf{X} \mathbf{1}_{1} \geq t \mathbf{1}_{1}^{\mathrm{T}} \mathbf{X} \mathbf{1}_{2}\right\},
$$

are convex. We can now solve Problem (5) efficiently using the well-known bisection method. 


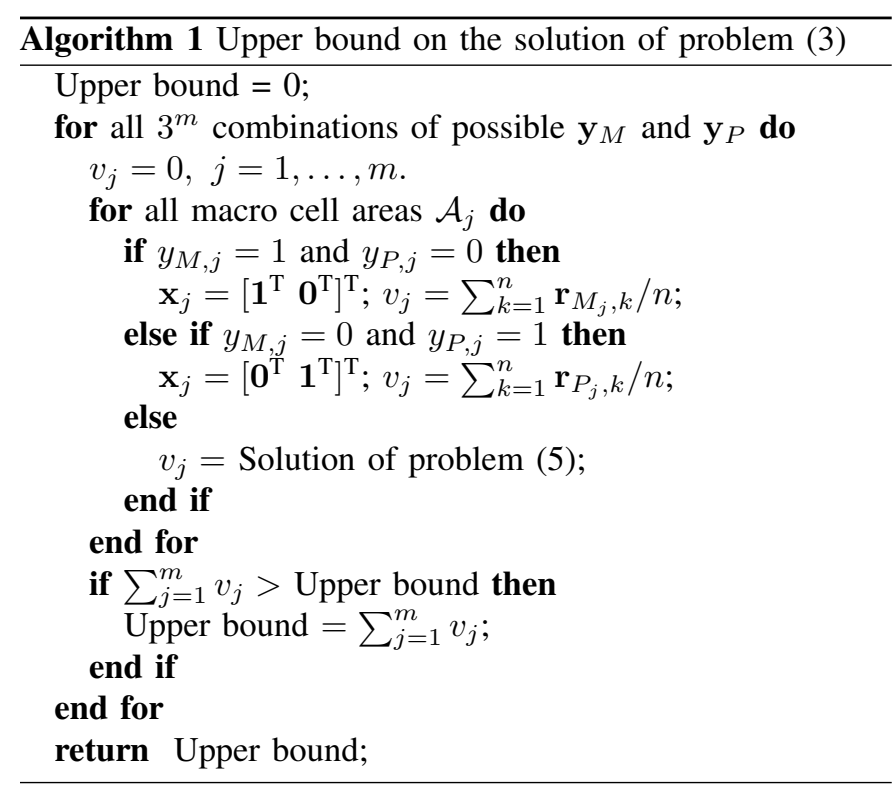

We summarize our method to find an upper bound on the solution of Problem (3) in Algorithm (1).

\section{Dynamic range heuristic}

The users are first sorted according to the difference in the power they received from the nodes in their macro cell area: $\Delta \hat{\sigma}_{j k}=\hat{\sigma}_{P_{j}, j k}-\hat{\sigma}_{M_{j}, j k}$. We then compute the sum rate achieved by $m n+1$ different cell associations, starting with the initial state where all users are associated with their macro node. We associate the UE with the largest $\Delta \hat{\sigma}_{j k}$ with its pico node, compute the resulting sum rate and repeat this process until all UEs are with their respective pico node. We select the cell association providing the largest sum rate. This heuristic has a complexity linear in the number of UEs. Numerical evaluations in Section V suggest that it is close to optimal.

\section{Numerical ANALysis}

To evaluate how close the dynamic range heuristic can approach the upper bound, the achievable sum rate is calculated for several user drops in a simplified radio network composed of 3 macro nodes with a Tx power of $40 \mathrm{~W}$. One pico node using a Tx power of $1 \mathrm{~W}$ is dropped in each macro cell area which has a radius of $167 \mathrm{~m}$. Each node operates with a $5 \mathrm{MHz}$ bandwidth. Fig. 1 illustrates the considered scenario.

As proposed in [8], the average channel gain between the macro node of the area $\mathcal{A}_{i}$ and a user $k$ in $\mathcal{U}_{j}$ is given by $g_{M_{i}, j k}=-\left(128.1+37.6 \log \left(d_{M_{i}, j k}\right)\right)$, while the average channel gain between the pico node of $\mathcal{A}_{i}$ and a user $k$ in $\mathcal{U}_{j}$ is $g_{P_{i}, j k}=-\left(140.7+36.7 \log \left(d_{P_{i}, j k}\right)\right) . d_{M_{i}, j k}$, resp. $d_{P_{i}, j k}$, denotes the distance between the user $k$ of $\mathcal{A}_{j}$ and the macro node, resp. the pico node, of $\mathcal{A}_{i}$ in $\mathrm{km}$. The users are dropped in a hotspot of $40 \mathrm{~m}$ radius around the pico node with a probability of $2 / 3$.

To produce Fig. 2 and Fig. 3, the level of the noise + outside cluster interference $\sigma_{n}$ has been varied from $-160 \mathrm{dBW}$ to $-40 \mathrm{dBW}$. For each simulated interference level, 200 drops of overall 10 users in the system have been performed. For

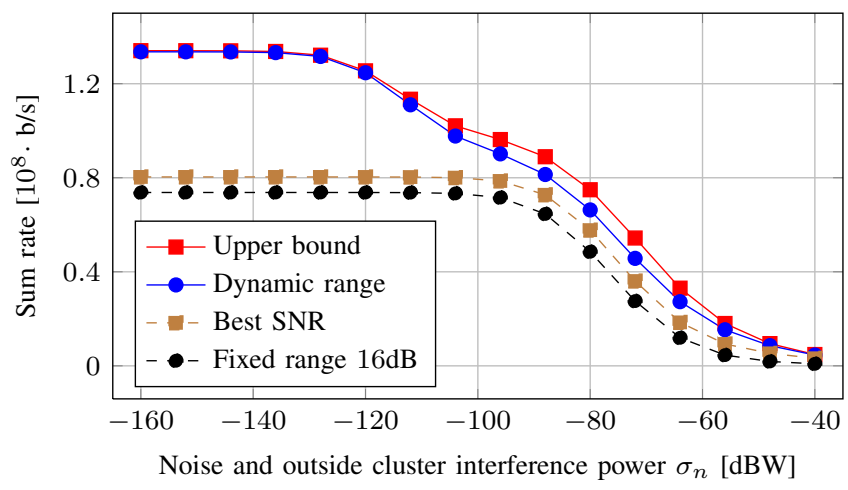

Fig. 2. UE sum rate for different cell associations.

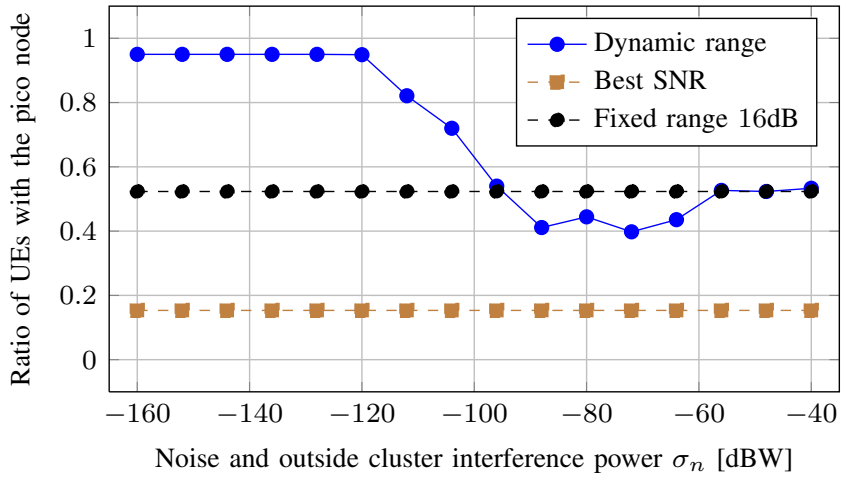

Fig. 3. Ratio of UEs with pico nodes for different cell associations.

each drop, the corresponding achievable rate for each user with different cell association heuristics has been measured.

We compare the best SNR heuristic, the fixed range heuristic and the dynamic range heuristic with each other, as well as with the upper bound. For the fixed range heuristic we choose a bias of $10 \log \left(\sigma_{M_{j}} / \sigma_{P_{j}}\right)=16 \mathrm{~dB}$.

In Fig. 2, the achievable sum rate calculated with different heuristics is shown for different $\sigma_{n}$. It can be seen that the dynamic range heuristic outperforms all the other heuristics and is very close to the upper bound. Consequently, the dynamic range heuristic is nearly optimal.

Looking at the pico user ratio for different interference levels in Fig. 3, it can be observed that dynamic range heuristic assigns almost no user to the macro node at low to medium interference level coming from outside $\mathcal{S}$. Consequently, the load is very imbalanced between the macro and pico nodes, but this ensures that no additional interference is created by the macro node. Such kind of cell association is possible in the simulated scenario, since user performance is not noiselimited but interference-limited. When the interference level from outside $\mathcal{S}$ increases, adding interference from the macro nodes does not affect the SINR so much anymore and it is worth balancing the load between the macro and pico nodes. In this case, users are indeed equally shared among the macro and pico nodes. The fixed range achieves a balanced load but does not improve the sum rate.

\section{Simulation Results for an LTE HetNet}

The three heuristics for the cell association have been implemented in a dynamic radio network simulator modeling 


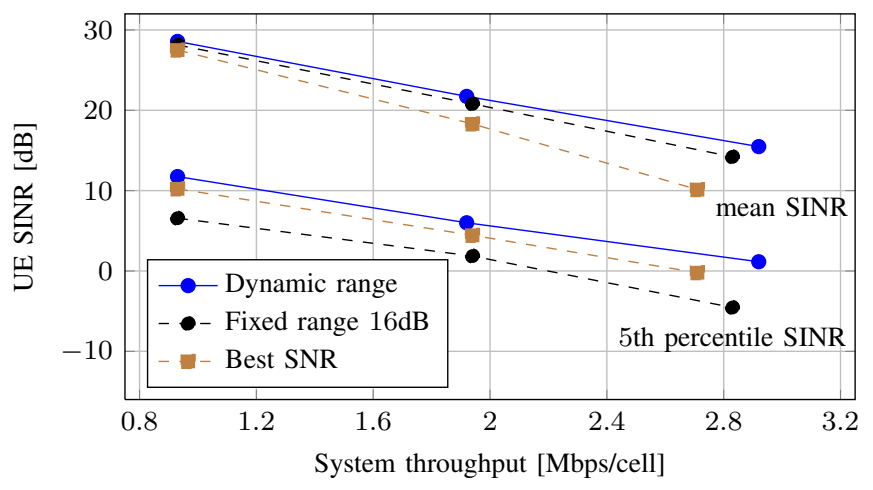

Fig. 4. Cell-edge and mean user SINR for different cell associations.

OFDM transmission with one antenna at the transmitters and receivers. The simulated $5 \mathrm{MHz}$ FDD LTE network is larger than in Section V. It consists of three sites with three macro cells per site and an inter-site distance of $500 \mathrm{~m}$. A HetNet with hotspots as defined in the configuration $4 b$ of [8] is considered. One pico node and 30 users are placed in each macro cell area. The Tx power of the macro and pico nodes are the same as previously. $\sigma_{n}$ was set to $-128 \mathrm{dBW}$. All cells operate at a carrier frequency of $2 \mathrm{GHz}$. The pathloss model 1 specified in [8] and cited previously is used.

The link adaptation is ideal and enables the selection between QPSK, 16QAM and 64QAM modulation schemes and several channel coding rates. Perfect channel estimation is assumed. A Round Robin scheduler allocates evenly resources to the active users. A FTP based traffic model is simulated as described in [8]. Users are created according to a Poisson process, they transfer a file of $500 \mathrm{kByte}$ and disappear from the system. Several user arrival rates were simulated to obtain different values for the system throughput.

Fig. 4 and Fig. 5 show the user SINR and the user throughput that were measured during simulations considering the available modulation and coding schemes, potential packet decoding errors and retransmissions. The 5th percentile user SINR, also called cell-edge user SINR, for different heuristics is represented in the lower part of Fig. 4, while the three curves in the upper part refer to the average user SINR. Fig. 4 shows that the best SNR cell association ensures a good user SINR at the cell-edge and a balanced user SINR distribution. By contrast, lower values for the cell-edge user SINR are observed for the fixed range heuristic, since users can be assigned to the node which does not have the largest average received signal strength. However, a larger average user SINR can be observed, which is due to the more balanced load share. The pico user ratio reaches $50 \%$ instead of $20 \%$ with the best SNR heuristic. Consequently users finish their transmission faster and the nodes are less active during the simulation time, yielding a reduced overall interference level and thus a larger average user SINR. As expected, the dynamic range heuristic achieves an improvement in both the cell-edge and average user SINR. This comes from the optimization problem in which both the interference and the user share among the nodes play a role. The pico user ratio varies from $44 \%$ to $66 \%$ for the three considered system loads.

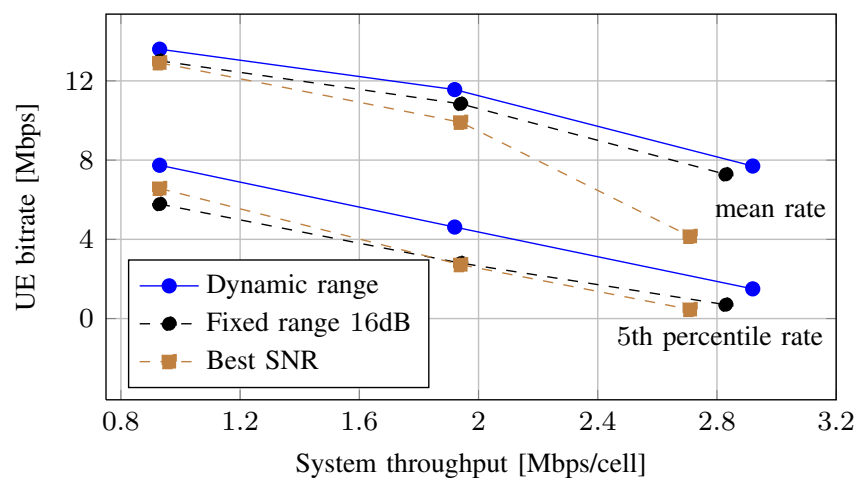

Fig. 5. Cell-edge and mean user bitrate for different cell associations.

From Fig. 5, it can be seen that the fixed range heuristic is beneficial for the user bitrate at medium to high system load compared to the best SNR association, even if the cell-edge user SINR is lower. The effect of lower SINR for these users is compensated by the larger amount of resources available for their transmission. The packet transmission of good users is faster in case of the fixed range and dynamic range heuristics, which leaves more resources available for the remaining users. Fig. 5 also shows that the dynamic range heuristic outperforms both the best SNR and fixed range associations in all considered system load values. This heuristic handles well the trade-off between the interference and user share among the nodes. The dynamic range provides a gain of $70 \%$ and $17 \%$ in the cell-edge user rate and mean user rate respectively, for medium system load, compared to the best SNR heuristic.

\section{CONCLUSION}

In this work we have presented a theoretical framework to study the impact of cell association on the downlink performance of heterogeneous networks. We have derived an upper bound on the achievable sum rate and proposed a dynamic range extension algorithm, which outperforms the conventional cell association scheme and comes close to the theoretical limit. We have implemented the dynamic range extension in an LTE simulator and demonstrated its performance in a realistic scenario.

\section{REFERENCES}

[1] NTT-DOCOMO, "Performance of eICIC with Control Channel Coverage Limitation," 3GPP TSG RAN WG1 Meeting 61, R1-103264.

[2] A. Khandekar, N. Bhushan, J. Tingfang, and V. Vanghi, "LTE-Advanced: Heterogeneous networks," in European Wireless Conference, 2010.

[3] S. Das, H. Viswanathan, and G. Rittenhouse, "Dynamic load balancing through coordinated scheduling in packet data systems," in Proc. IEEE INFOCOM 2003, march-april 2003, pp. 786 - 796 vol.1.

[4] T. Bu, L. Li, and R. Ramjee, "Generalized proportional fair scheduling in third generation wireless data networks," in Proc. IEEE INFOCOM 2006, april 2006.

[5] K. Son, S. Chong, and G. Veciana, "Dynamic association for load balancing and interference avoidance in multi-cell networks," IEEE Trans. on Wireless Commun., vol. 8, pp. 3566 - 3576, July 2009.

[6] S. Parkvall, E. Dahlman, G. Jöngren, S. Landström, and L. Lindbom, "Heterogeneous network deployments in LTE," Ericsson Review, vol. 90, pp. 34-38, 2011.

[7] S. Boyd and L. Vandenberghe, Convex optimization. Cambridge university press, 2008 (sixth printing).

[8] "3GPP TR36.814, Further advancements for E-UTRA physical layer aspects." 